\title{
Miscarriage after Sonographic Confirmation of an Ongoing Pregnancy in Women with Moderate and Severe Obesity
}

\author{
Vicky O'Dwyer Bernadette Monaghan Chro Fattah Nadine Farah \\ Mairead M. Kennelly Michael J. Turner \\ UCD Centre for Human Reproduction, Coombe Women and Infants University Hospital, \\ Dublin, Ireland
}

\author{
Key Words \\ Maternal obesity $\cdot$ Miscarriage $\cdot$ BMI
}

\section{Abstract}

Objective: To compare the incidence of spontaneous miscarriage in women with moderate to severe obesity to that in women with a normal BMI after sonographic confirmation of the foetal heart rate in the first trimester. Methods: Women were enrolled in a prospective observational study at their convenience in the first trimester after an ultrasound confirmed an ongoing singleton pregnancy with foetal heart activity present. Maternal height and weight were measured digitally and BMI was calculated. Results: In the 3,000 women enrolled, the miscarriage rate overall was $3.9 \%(n=117)$. The mean gestational age at enrolment was 11.1 weeks. In the class $2-3\left(\mathrm{BMI}>34.9 \mathrm{~kg} / \mathrm{m}^{2}\right)$ obese primigravidas the miscarriage rate was $11.3 \%(n=8)$ compared with $2.7 \%(n=24)$ in the normal BMI category $(p=0.003)$, and $3.7 \%$ $(n=5)$ in the class 1 obese category (not significant). In multigravidas, there was no increased rate of miscarriage among class 2-3 obese women compared with multigravidas in the normal BMI category. The mean body composition values showed that primigravidas who miscarried had both increased fat and fat-free masses compared with those who did not, but multigravidas who miscarried had a similar fat mass and fat-free mass with those who did not. Conclusions: In women with sonographic evidence of foetal heart activity in the first trimester, the rate of spontaneous miscarriage is low. It was increased in moderate to severely obese primigravidas, but was not increased in other obese women compared to women in the normal BMI category. 
O'Dwyer et al.: Miscarriage after Sonographic Confirmation of an Ongoing

Pregnancy in Women with Moderate and Severe Obesity

\section{Introduction}

Spontaneous miscarriage is the commonest complication in pregnancy and may occur in up to one fifth of clinical pregnancies. Although the majority of miscarriages are due to genetic abnormalities, the experience can be very distressing for the woman and her family.

Based on the World Health Organization (WHO) classification, obesity is defined as BMI $>29.9 \mathrm{~kg} / \mathrm{m}^{2}$. Maternal obesity is associated with an increase in pregnancy complications [1]. There is also evidence that the levels of maternal obesity are increasing in developed countries. In Great Britain and Ireland, nearly one fifth of women booking for antenatal care are obese $[2,3]$. Based on the WHO sub-categorisation of obesity, a recent UK national audit of pregnant women found that $5.0 \%$ had class 2 or moderate obesity and $2.0 \%$ had class 3 or severe obesity [3].

It has been reported that maternal obesity is associated with an increased risk of spontaneous miscarriage after spontaneous and assisted conception [4]. However, in a recent study of 1,200 women, we found that in women with sonographic evidence of foetal heart activity in the first trimester, the rate of spontaneous miscarriage was low and not increased in women with a BMI $>29.9 \mathrm{~kg} / \mathrm{m}^{2}$ compared to women in the normal BMI category [5]. However, the numbers reported previously were too small to allow any meaningful analysis of the results by WHO BMI obesity sub-categories. Therefore, we continued the research, and the purpose of this study was to analyse the miscarriage rate by WHO obesity subcategory and by parity. We also used advanced bioelectrical impedance analysis (BIA) to compare maternal fat levels in those women who miscarried with those who did not [2].

\section{Participants and Methods}

A prospective observational study was conducted in a university teaching hospital with an annual delivery rate of approximately 9,000 babies weighing $500 \mathrm{~g}$ or more. One in 8 pregnant women in the Republic of Ireland from all socio-economic groups attends our hospital, and admissions are not differentiated on the basis of social status, private health insurance or clinical risk. Women were enrolled at their convenience in the first trimester after an ultrasound confirmed an ongoing pregnancy with foetal heart activity present. To minimise confounding variables, the study was confined to white European women with a singleton pregnancy. Written consent was obtained.

The hospital practice is to perform the dating ultrasound before measurement of BMI and before the computerisation of the clinical history. Women who had already miscarried were excluded. A diagnosis of miscarriage was confined to women with a history of amenorrhoea and a positive pregnancy test who miscarried spontaneously with a foetal weight $<500 \mathrm{~g}$.

Maternal height was measured using a Seca wall-mounted digital meter stick to the nearest centimetre with the woman standing in her bare feet. Maternal weight was measured digitally and BMI calculated. Body composition was measured by segmental multi-frequency BIA (Tanita MC-180; Tanita, Tokyo, Japan). BIA measures the resistance of body tissues to a small electrical current. The current passes faster through fat-free mass and water than through fat mass. A figure of $0.5 \mathrm{~kg}$ was allowed for light clothing when measuring weight. The woman was asked to stand on the Tanita machine, to take the two handles and to hold them by her side. The measurement took $15 \mathrm{~s}$. Body composition measurements included fat mass, fat-free mass, visceral fat level, total body water and bone mass. We have previously shown that the measurement of maternal body composition in all three trimesters of pregnancy is reproducible [6]. The measurement is safe and quick to perform, making it useful for widespread clinical use. A previous study found that the mean maternal fat mass, fat-free mass and bone mass remained unchanged in the first trimester of pregnancy [2].

Clinical and socio-demographic details were collected for subsequent analysis. Statistical analysis was performed using SPSS version 18.0 (Chicago, IL, USA) and a p value $<0.05$ was considered significant. Fischer's test was used to calculate $\mathrm{p}$ values. The independent sample t-test was used to compare means. The study was approved by the Hospital's Research Ethics Committee. 
Table 1. Miscarriage rate analysed by parity and BMI category

\begin{tabular}{llll}
\hline $\begin{array}{l}\text { BMI category, } \\
\mathrm{kg} / \mathrm{m}^{2}\end{array}$ & $\begin{array}{l}\mathrm{n}=1,482) \\
<18.5\end{array}$ & $\begin{array}{l}\text { Multigravidas } \\
(\mathrm{n}=1,518)\end{array}$ & $\begin{array}{l}\text { Total } \\
(\mathrm{n}=3,000)\end{array}$ \\
\hline $18.5-24.9$ & $2.7 \%(24 / 49)$ & $5.0 \%(1 / 20)$ & $4.3 \%(3 / 69)$ \\
$25.0-29.9$ & $4.3 \%(15 / 351)$ & $4.5 \%(32 / 717)$ & $3.6 \%(56 / 1,583)$ \\
$30.0-34.9$ & $3.7 \%(5 / 135)$ & $2.2 \%(4 / 178)$ & $2.9 \%(9 / 313)$ \\
$>34.9$ & $11.3 \%(8 / 71)^{*}$ & $2.9 \%(4 / 136)$ & $5.8 \%(12 / 207)$ \\
Total & $3.6 \%(55 / 1,482)$ & $4.2 \%(63 / 1,518)$ & $3.9 \%(117 / 3,000)$
\end{tabular}

${ }^{*} \mathrm{p}=0.003$. All other comparisons not significant.

\section{Results}

Of the 3,000 women enrolled, there were 1,482 primigravidas and 1,518 multigravidas. $17.3 \%$ of the women were obese. The mean ( \pm SD) age of the women enrolled was $28.3 \pm$ 5.6 years. The overall miscarriage rate was $3.9 \%(\mathrm{n}=117)$. The mean gestational age at enrolment was $11.1 \pm 2.1$ weeks for women who did not miscarry $(n=2,883)$ compared with $10.3 \pm 2.5$ weeks for those who did $(\mathrm{n}=117)$.

The age at which women left full-time education was used as a surrogate marker for socio-economic status. There was no difference in the mean age at or when women who miscarried left full-time education compared with those who did not $(19.1 \pm 3.3$ years vs. $19.9 \pm 4.2$ years; $p=0.56$ ). There were no women with a history of uterine abnormality or cervical incompetence in the group who miscarried. It is hospital policy to only refer women with recurrent miscarriage or second trimester pregnancy loss for investigation. There was no difference in the number of women who smoked in the group who miscarried compared with those who did not (22.8\% vs. $22.9 \%$; $=0.31$ ). There was also no difference in the incidence of smoking in women who had a normal BMI (22.1\%), were mildly obese $(25.5 \%)$ or moderate to severely obese (22.6\%).

The miscarriage rates are analysed by the WHO BMI categories and parity (table 1). Overall, the miscarriage rate in obese women was $4.0 \%(n=21)$ compared with $3.6 \%$ $(\mathrm{n}=56)$ in the normal BMI category (not significant), which is similar to the results we previously published [5]. However, when analysed by subcategories we found that the miscarriage rate was higher $(11.3 \%)$ in primigravidas with moderate to severe obesity $(p=0.003)$, but that there was no increase in primigravidas with mild obesity or in multigravidas irrespective of BMI category compared with the miscarriage rates in the normal BMI category.

The mean body composition values of primigravidas and multigravidas are shown in table 2. This shows that primigravidas who miscarried had increased fat and fat-free masses compared with those who did not; but multigravidas who miscarried had similar fat masses and fat-free masses compared with those who did not. This is consistent with the results in table 1.

Of the primigravidas with moderate to severe obesity, the mean age was $30.8 \pm 5.2$ years, and $21.7 \%$ were $>35$ years of age. Of the 8 women in this group who miscarried, the mean age was $31.1 \pm 4.8$ years, and there was only 1 woman $>35$ years of age. This indicates that increased age does not explain the higher incidence of miscarriage in primigravidas with class $2-3$ obesity. 
O'Dwyer et al.: Miscarriage after Sonographic Confirmation of an Ongoing

Pregnancy in Women with Moderate and Severe Obesity

Table 2. Comparison of mean body composition in primigravidas and multigravidas who miscarried with those who did not

\begin{tabular}{lllllll}
\hline & $\begin{array}{l}\text { Primigravidas } \\
\text { who miscarried } \\
(\mathrm{n}=55)\end{array}$ & $\begin{array}{l}\text { Primigravidas } \\
\text { who did not } \\
\text { miscarry } \\
(\mathrm{n}=1,427)\end{array}$ & $\mathrm{p}$ value & $\begin{array}{l}\text { Multigravidas } \\
\text { who miscarried } \\
(\mathrm{n}=62)\end{array}$ & $\begin{array}{l}\text { Multigravidas } \\
\text { who did not } \\
\text { miscarry } \\
(\mathrm{n}=1,451)\end{array}$ & $\mathrm{p}$ value \\
\hline $\mathrm{BMI}, \mathrm{kg} / \mathrm{m}^{2}$ & 26.7 & 24.7 & 0.002 & 26.5 & 25.5 & 0.31 \\
Fat mass, kg & 24.9 & 21.2 & 0.006 & 24.1 & 22.3 & 0.36 \\
Fat-free mass & 47.0 & 45.3 & 0.04 & 46.8 & 47.6 & 0.35 \\
Visceral fat (level) & 4.2 & 3.3 & 0.005 & 4.4 & 4.0 & 0.33 \\
\% Body fat & 31.4 & 29.8 & 0.11 & 32.4 & 30.9 & 0.11 \\
\hline
\end{tabular}

Of the primigravidas with class 2-3 obesity who miscarried, 1 woman had a history of infertility secondary to polycystic ovarian syndrome (PCOS), and 1 had a history of recurrent miscarriage. Three women in this subgroup previously miscarried. One of the women had a history of type 2 diabetes mellitus. None of the 8 women had a history of thyroid disease or thrombophilia.

\section{Discussion}

In women with sonographic evidence of foetal heart activity in the first trimester, the rate of spontaneous miscarriage was low as expected. We found that the spontaneous miscarriage rate was increased in primigravidas with class 2-3 obesity, but was not increased in other obese women compared to women in the normal BMI category. Measurement of body composition showed that in primigravidas, but not in multigravidas who miscarried, adiposity levels were higher compared with women who did not miscarry.

A limitation of this study is that our findings apply only to women with sonographic confirmation of foetal heart rate activity in the first trimester of pregnancy. Women who are clinically pregnant may miscarry in a community setting or may miscarry before a scheduled ultrasound assessment. Also, many women who have PCOS are obese and have oligomenorrhoea. Thus, it is challenging to study prospectively all clinical miscarriages in women where the pregnancy has been accurately dated, and where maternal adiposity is measured rather than self-reported. This explains the paucity of studies on this subject to date, particularly outside an infertility treatment setting.

The number of women with moderate to severe obesity may appear small, but it is similar to recent reports [3]. Also previous studies based on self-reporting of weight may have assigned moderate to severely obese women to the mild obesity category [15-17].

Miscarriage rates are inversely related to gestational age [7]. A foetal heart can be detected on transvaginal ultrasound as early as 6 weeks gestation or when a foetal pole reaches $5 \mathrm{~mm}$ in size. The risk of miscarriage is $5 \%$ once a foetal heart has been detected on ultrasound scan [9]. Miscarriages also increase with increasing maternal age [8]. This is due to poorer quality oocytes and resulting chromosomal abnormalities. Chromosomal abnormalities are the commonest cause of miscarriage. Higher rates of miscarriage have been described among obese women requiring assisted reproduction. Increased rates of miscarriage occur in obese women with PCOS and after assisted reproduction [10,11]. In a Finnish 
study, the miscarriage rate was found to be higher at the extremes of weight compared with normal-weight women [12]. In a study examining the probability of pregnancy after assisted reproduction the odds ratio was 0.73 for class 1 obese women and 0.5 for class 2-3 obese women, compared with women in the normal BMI category [13].

Recurrent miscarriage is defined as three consecutive miscarriages. It affects $1 \%$ of couples. In a study of 491 women with a history of recurrent miscarriage, the miscarriage rates in subsequent pregnancies were higher in obese women compared with normal-weight women [4]. In another study, the risk of recurrent miscarriage was four times higher in obese women compared with normal-weight controls. Diabetes mellitus is a known cause of recurrent miscarriage but, in this study the prevalence was low in obese women and it did not explain their higher risk of miscarriage [10].In our study, BMI was measured and not based on self-reporting. Self-reporting has been shown to be inaccurate and can lead to exaggeration of the risks of obesity [15-17]. The hospital policy of performing a dating scan at the woman's booking visit allowed us to date the pregnancy and detect a foetal heart beat. The limitation of our study is that the number of patients who are morbidly obese is relatively small. Our hospital is, however, a large teaching hospital, and our patient population is representative of the Irish population.

Obesity is a modifiable risk factor for infertility, miscarriage and adverse pregnancy outcomes. The National Institute of Health has recently addressed the issue of gestational weight gain but the issue of optimising weight loss among obese women of reproductive age needs to be highlighted. Class 2-3 obese women should be advised to lose weight before conception to minimise the risk of miscarriage, and indeed, other pregnancy complications.

\section{Disclosure Statement}

There are no conflicts of interest the authors need to disclose.

\section{References}

1 Barry S, Fattah C, Farah N, Broderick V, Stuart B, Turner MJ: The growing challenge of maternal obesity. Ir Med J 2009:102; 5-6.

2 Fattah C, Farah N, Barry SC, O'Connor N, Stuart B, Turner MJ: Maternal weight and body composition in the first trimester of pregnancy. Acta Obstet Gynecol Scand2010;89:952-955.

3 Centre for Maternal and Child Enquiries: Maternal Obesity in the UK: Findings from a National Project. 2010. www.oaa-anaes.ac.uk/assets/_managed/editor/File/CMACE/CMACE_Obesity_Report_2010_Final\%20 for\%20printing.pdf.

4 Metwally M, Ong KJ, Ledger WL, Li TC: Does high body mass index increase the risk of miscarriage after spontaneous and assisted conception? A meta-analysis of the evidence. Fertil Steril 2008;90:714-726.

- 5 Turner MJ, Fattah C, O'Connor N, Farah N, Kennelly M, Stuart B: Body mass index and spontaneous miscarriage. Eur J Obstet Gynecol 2010;151:169-170.

6 Hogan JL, Farah N, O'Connor N, Kennelly MM, Stuart B, Turner MJ: Bioelectrical impedance analysis and maternal body composition: reproducibility of bioelectrical impedance when analysing maternal body composition. Int J Body Comp Res 2011 (in press).

- 7 Brigham SA, Conlon C, Farquharson RG: A longitudinal study of pregnancy outcomes following recurrent miscarriage. Hum Reprod 1999;14:2868-2871.

8 Heffner LJ: Advanced maternal age-how old is too old? N Engl J Med 2004;351:1927-1929.

- 9 VanLeeuwen I, Branch DW, Scott JR: First trimester ultrasonography findings in women with a history of recurrent pregnancy loss. Am J Obstet Gynecol 1993;168:111-114.

10 Lashen H, Fear K, Sturdee DW: Obesity is associated with increased risk of first trimester and recurrent miscarriage: matched case-control study. Hum Reprod 2004;19:1644-1646.

-11 Bellver J, Rossal LP, Bosch E, Zuniga A, Corona JT, Melendez F, Gomez E, Simon C, Remohi J, Pellicer A: Obesity and the risk of spontaneous abortion after oocyte donation. Fertil Steril 2003;5:1136-1140. 
12 Veleva Z, Tiitinen A, Vilska S, Hyden-Granskog C, Tomas C, Martikainen H, Tapanainen JS: High and low BMI increase the risk of miscarriage after IVF/ICSI and FET. Hum Reprod 2008;23:878-884.

13 Wang JX, Davies MJ, Norman RJ: Body mass and probability of pregnancy during assisted reproduction treatment: retrospective study. BMJ 2000;321:1320-1321.

14 Wang JX, Davies MJ, Norman RJ: Obesity increases the risk of spontaneous abortion during infertility treatment. Obes Res 2002;10:551-554.

15 Shields M, Gorber SC, Tremblay MS: Estimates of obesity based on self-report versus direct measures. Health Rep 2008;19:61-76.

-16 Fattah C, Farah N, O’Toole F, Barry S, Stuart B, Turner MJ: Body mass index (BMI) in women booking for antenatal care: comparison between self-reported and digital measurements. Eur J Obstet Gynecol Reprod Biol 2009;144:32-34.

17 Shields M, Gorber SC, Tremblay MS: Estimates of obesity based on self-report versus direct measures. Health Rep 2008;19:61-76. 